\title{
Strates
}

STRATES Matériaux pour la recherche en sciences sociales

6 | 1992

La question de l'environnement : naissance d'un débat en Pologne

\section{Région, régionalismes et aménagement}

\section{Bernard Barraque}

\section{(2) OpenEdition \\ Journals}

Édition électronique

URL : http://journals.openedition.org/strates/3523

DOI : $10.4000 /$ strates.3523

ISSN : $1777-5442$

Éditeur

Laboratoire Ladyss

Édition imprimée

Date de publication : 31 mars 1992

ISSN : 0768-8067

Référence électronique

Bernard Barraque, «Région, régionalismes et aménagement », Strates [En ligne], 6 | 1992, mis en ligne le 22 novembre 2007, consulté le 21 décembre 2020. URL : http://journals.openedition.org/strates/

3523 ; DOI : https://doi.org/10.4000/strates.3523

Ce document a été généré automatiquement le 21 décembre 2020.

Tous droits réservés 


\title{
Région, régionalismes et aménagement
}

\author{
Bernard Barraque
}

1 La région est maintenant une institution administrative établie en France, et d'aucuns pensent que son rôle va encore s'affirmer dans le cadre de l'unité européenne. Mais la région, pour faire quelle économie et quelle politique ? En France au moins, le terme est ambigu, car il est mobilisé contradictoirement par les partisans de la modernisation et par ceux de la défense du patrimoine, par les «aménageurs »et les «ménageurs $»^{1}$. L'hypothèse centrale de notre réflexion est que l'analyse du débat qui s'est développé entre ces deux courants permet de mettre en lumière la lente transformation du regard que la société porte sur elle-même, et de la façon d'y faire de la politique. Pour tenter de le montrer, il faut remonter assez loin dans la durée, et en particulier au régionalisme du début du siècle.

2 Mais ne serait-ce que depuis 1945 , le terme de région a été utilisé de façons très diverses: l'aménagement du territoire et l'action régionale, intitulé de la DATAR, rappellent les métropoles d'équilibre, les armatures urbaines et toute l'approche de spatialisation, d'imposition aux territoires de la planification française. La région a été incontestablement liée aux grands équipements conçus dans une démarche aussi technocratique qu'abstraite, mais elle n'a pas été que cela, comme en témoignent les parcs régionaux : en les créant en 1967, la DATAR visait à faire participer les habitantsagriculteurs des parcs à une gestion pluri-objectifs de ces espaces combinant la protection de la nature, le maintien de l'activité agricole et la mise en valeur touristique. De son côté, le terme même de métropole d'équilibre appelle en partie une notion de modération, de ménagement du patrimoine, en s'opposant à la fois au gigantisme de la capitale et à la désertification du reste de la France (Robic, 1989a).

La région, c'est aussi la base territoriale proposée par le général de Gaulle pour assurer une meilleure participation de la majorité des citoyens à la gestion du pays : projet régional associé à la création du "sénat économique et social ». Aurait-il vu le jour si le général n'avait pas mis sa personne dans la balance? Plus encore que le «non » au référendum de 1969, c'est la fin de la grande période de planification qui a fait reculer 
le thème régional ou qui l'a dissous dans une flambée régionaliste. Celle-ci prenait peut-être un tour d'autant plus vif au plan culturel que la région ne semblait pas encore avoir percé sur le plan socio-économique au début des années soixante-dix. Pierre Grémion (1976) montre bien comment l'articulation traditionnelle entre notables et fonctionnaires territoriaux du niveau départemental a résisté, et par là même a limité l'importance prise par la région. Mais pour autant, cette résistance du "pouvoir périphérique" soit-elle être assimilée à une victoire du ménagement sur l'aménagement? Il se pourrait au contraire qu'elle ait une fois de plus fait prévaloir un modèle d'organisation jacobin et centralisateur, ou tout au moins un «antagonisme réglé » qui met en valeur le centre, au détriment d'une auto-organisation décentralisatrice plus en prise sur les réalités locales² (Marié, 1986, Gaudin 1986).

4 La région paraît être une notion floue, car elle renvoie tantôt à un redécoupage territorial (et d'ailleurs, soit supra départemental, soit plus local que le département), tantôt à un projet de réforme sociale ou politique ; elle est utilisée aussi bien par les traditionalistes que par les modernistes. Ce qui ajoute encore à la confusion, c'est que les volontés de décentralisation régionale ont été portées successivement par différents partis et courants de pensée, mais justement avec des arrière-pensées politiques d'établissement de contre-pouvoirs. Et, dès que ces partis ont conquis la majorité nationale, ils ont mis au second plan puis abandonné leur projet régional, devenu moins « opportun » : les radicaux au début du siècle, la démocratie chrétienne en 1945, et même dans une moindre mesure le parti socialiste tout récemment.

Une chose semble assez claire, toutefois : la région est associée à une façon de regarder l'espace. Les géographes ont bien sûr joué là un rôle essentiel. Il convient cependant d'insister ici sur l'importance du « local » dès le début du siècle.

Les études locales en France

6 Une piste s'ouvre avec un ouvrage figurant dans la bibliographie de Robert Auzelle (1963), consacré à la « pédagogie active " par l'étude du milieu et au rôle joué en cette matière par l'urbaniste écossais Patrick Geddes. Car Mabel Barker (1931), en évoquant l'étude du milieu à des fins pédagogiques dans d'autres pays que l'Angleterre, mentionne la création à Paris, en 1911, de la "Société des études locales", par le ministre de l'Instruction publique de l'époque, Maurice Faure, ainsi que les travaux sur le régionalisme de Jean Charles-Brun ${ }^{3}$. Or ce sont deux noms qui figurent dans une nébuleuse intellectuelle où l'on trouve aussi bien les défenseurs des sites que les premiers promoteurs de l'aménagement des villes, notamment Charles Beauquier et le Vicomte Cornudet, et bon nombre de membres d'un courant politique assez lâche qui se centrait sur la Fédération régionaliste, mais qui s'étendait aussi bien à l'école de géographie humaine de Vidal de la Blache qu'aux disciples de Proudhon.

7 En découvrant l'existence de cette Société des études locales dans un ouvrage consacré à la remise en cause de la pédagogie autoritaire et "statique », on aurait pu imaginer trouver en France, dès le début du siècle, un mouvement de type "écologiste », antiautoritaire, largement précurseur par rapport au "pouvoir culturel» des «animateurs» que Joffre Dumazedier appelle de ses vœux pour aboutir à une « civilisation des loisirs» (1967 et 1976).

Dans cette « civilisation ", l'animation culturelle jouerait le rôle qu'on désespère de voir jouer par l'école de la République : l'élévation de tous au rang de citoyen pouvant participer pleinement à la cité. Et la pratique studieuse du milieu naturel doit y jouer un rôle important. 
9 En fait, la lecture des rares archives qui subsistent ${ }^{4}$ conduit à une appréciation beaucoup plus mesurée du rôle de cette société. Organisée en sections départementales, elle devait réunir autour de l'inspecteur d'Académie les enseignants volontaires d'histoire et de géographie, l'archiviste paléographe, les représentants des sociétés savantes, les abbés cultivés, etc., afin de "mêler intimement l'enseignement de la géographie et de l'histoire locales à celui de la géographie et de l'histoire nationales, en puisant le plus possible les exemples dans le milieu même où les élèves résident, qu'ils connaissent et qu'ils aiment $\|^{5}$. La motivation avancée était entre autres celle de fonder l'attachement à la Grande patrie (la France) par la mise en valeur de l'enracinement dans le sol natal. C'est un thème ruskinien qui était alors avancé par les défenseurs du patrimoine historique, pittoresque, légendaire, par les défenseurs des sites, les régionalistes (CrosMayrevieille, 1907). Toutefois, c'est à notre avis d'abord par politesse que Mabel Barker mentionne cette approche patrimoniale. Car à lire les bulletins émanant des sections de cette société, on cherche en vain un élément de pédagogie active, fondée sur la découverte du milieu par l'enfant. Les travaux rassemblés sont plutôt des travaux d'érudition très spécialisés pouvant intéresser un public restreint d'autodidactes et de membres adultes de sociétés savantes. De surcroît, le fait que l'initiative ait été prise d'en haut et qu'elle se soit déclinée ensuite par département (ou par académie scolaire, ce qui revient au même) était tout à fait contradictoire avec l'idée même du " pays ", du " local », de la petite région, dans la mesure où ces derniers sont définis "par en bas ", alors que le département était alors le point d'ancrage du jacobinisme.

Il n'en reste pas moins que ces tentatives de régionalisme scolaire ont été réelles, et qu'elles se sont poursuivies au-delà de la deuxième guerre mondiale ${ }^{6}$. On ne peut alors manquer de se demander quel lien elles ont pu avoir avec l'audience de l'école de géographie française fondée elle aussi sur l'étude du milieu local (Berdoulay, 1981). D'autant plus que Vidal de la Blache lui-même, le chef de file de l'école, était un régionaliste convaincu. Il est l'auteur d'un projet de réforme régionale ${ }^{7}$, et il accepta de présider en novembre 1913 la leçon d'ouverture d'un cours consacré au redécoupage de la France, intitulée Pourquoi le régionalisme, prononcée par Raymond Lizop au Collège libre des sciences sociales. Cet auteur sera plus tard responsable, avec J. Charles-Brun, du découpage de la France en régions pendant le régime de Vichy (Lizop, 1938).

Le régionalisme au début du siècle

11 La veille de la première guerre mondiale fut la période faste d'un certain régionalisme que nous voudrions évoquer ici. Aristide Briand était devenu président du Conseil en 1910 notamment sur la promesse d'une réforme régionale, qui ne put être mise en œuvre à cause de la guerre.

Or une annexe du livre de J. Charles-Brun montre que c'était l'aboutissement d'un mouvement de longue durée, mais qui prenait de l'ampleur : de 1850 à 1911, il a recensé 21 projets de réforme régionale émanant de l'initiative privée et 7 émanant de l'initiative parlementaire, dont respectivement 13 et 2 dans les dix premières années du $\mathrm{xx}^{\mathrm{e}}$ siècle ${ }^{8}$. La majorité de ces projets conservaient les départements qu'ils regroupaient en régions, espérant voir les premiers dépérir plus tard; 12 projets supprimaient le niveau départemental ; la plupart se focalisaient en fait sur l'identification de capitales régionales.

$13 \mathrm{~J}$. Charles-Brun était le très actif secrétaire de la Fédération régionaliste, fondée en 1900. Il était folkloriste et romancier, ami personnel de F. Mistral, mais il était en même temps le secrétaire de la Société des amis de Proudhon. Dans son ouvrage, il 
montre que le régionalisme attirait alors un très large éventail politique : il rappelle dans l'avant-propos que le mot régionalisme permettait de réunir temporairement au moins les partisans de la décentralisation, nombreux comme en témoigne le succès du « programme de Nancy » (1865), et les fédéralistes, provincialistes, etc. - c'est-à-dire de réunir les tenants d'un mouvement descendant et ceux d'une reconstruction de l'État par le bas. Charles Maurras prétendait avoir rencontré le mot de régionalisme dès 1874 sous la plume d'un folkloriste provençal' ${ }^{9}$, mais J. Charles-Brun estime plus raisonnable de dater son émergence de la fin du siècle, avec la création en 1895 par Charles Beauquier ${ }^{10}$ de la Ligue nationale pour la décentralisation. L'année suivante, Jules Méline crée le Crédit agricole, et c'est certainement par le goût du président du Conseil et du « lobby agricole » pour le régionalisme que les caisses locales du Crédit agricole se sont appelées « régionales » alors qu'elles se sont finalement développées sur une base départementale, à de très rares exceptions près. En l'occurrence, « régional » renvoyait là à une entité du genre " pays » : la ville, son hinterland et ses notables... À cette même époque encore, l'abbé Lemire créait dans le Nord la «Ligue du coin de terre et du foyer ", dans une perspective de retour à la terre. Charles Maurras côtoyait aussi les régionalistes, imaginant sans doute que les régions seraient peu ou prou les anciennes provinces ou généralités. Il devait d'ailleurs s'éloigner de ce courant qu'il trouvait trop timoré dans la remise en cause de la République jacobine. Maurice Barrès, plus connu aujourd'hui pour son patriotisme, était venu au régionalisme à partir de sa défense du socialisme municipal de Roubaix (1894). Pour lui, la région devait être un laboratoire d'expérimentations sociales, dont le pays entier tirerait les leçons à moindres frais.

Certains défenseurs d'un syndicalisme modéré comme J.Paul-Boncour étaient également partisans de la région, en tant que lieu de réorganisation des corps professionnels abolis par un siècle de jacobinisme (loi Le Chapelier, articles 290-291 du Code pénal). Dès la IIe République, les proudhoniens avaient souhaité cette réorganisation des compagnonnages (corporations amputées des patrons) sur des bases régionales. Ils se rapprochaient ainsi des positions de corporatistes catholiques comme La Tour du Pin. Enfin les syndicalistes positivistes (A. Deherme, M. Ajam) qui ont joué un rôle dans l'émergence des Universités populaires, ainsi que dans l'introduction du paritarisme au Conseil supérieur du travail, étaient également favorables à la région, tout comme le socialiste Millerand, qui fit passer plusieurs lois importantes lors de son association avec les radicaux et certains libéraux, lors du cabinet Waldeck-Rousseau au tout début du $\mathrm{xx}^{\mathrm{e}}$ siècle.

Charles Beauquier, député radical du Doubs, semble avoir joué un rôle central dans ce mouvement : à plusieurs reprises, il a fait voter des motions en faveur d'un découpage régional lors de congrès de son parti. Folkloriste, défenseur du patrimoine, il prit la tête de la première bataille juridique de défense d'un site naturel contre des producteurs d'électricité et le gagna (Barraqué, 1991), ce qui lui valut la présidence de la Société pour la protection des paysages de France (SPPF), créée en 1901. Il s'appuya sur celle-ci pour faire voter la première loi de protection des sites (1906). Il souhaitait également la création de parcs nationaux (proposition de loi de 1908). Il participa surtout vivement à la préparation de la loi sur les plans d'aménagement et d'extension des villes, qui fut votée en 1919. En 1900, il réussit à regrouper un certain nombre d'initiatives dans la Fédération régionaliste, qu'il coprésidait avec X. de Marcère et L. Xavier de Ricard ${ }^{11}$. C'est l'homme d'un compromis possible entre modernisation et défense du patrimoine ; comme pour la plupart des membres du Musée social, les deux vont ensemble et ce n'est probablement pas un hasard s'ils ont adopté le mot aménagement pour caractériser 
leur démarche : le mot même ne contient-il pas l'idée d'un ménagement? C'est au cours des trente glorieuses que l'emploi du mot a pris une connotation plus «frontale».

Pour eux comme pour Charles-Brun, le régionalisme se caractérise d'abord par sa "méthode pour cerner la diversité de la psychologie et de la géographie »"12. L'auteur renvoie d'ailleurs aux travaux de l'équipe de Vidal de la Blache, ainsi qu'à la Revue de synthèse historique. L'idée est d'expérimenter la diversité au sein du pays. On cesserait de tout faire comme au centre, et on pourrait innover localement. C'est ensuite une discipline de modération : "ne jamais négliger le facteur temps »; ne pas étouffer le sentimentalisme de la tradition sous le volontarisme du système, faire preuve de réalisme ; refuser les ruptures en considérant, "d'un point de vue évolutionniste, les déterminations physiques des individus d'une région $»^{13}$. Le régionalisme est un essai de conciliation entre la tradition et le progrès, entre l'individu et l'État, entre le particularisme et le patriotisme. C'est pourquoi il conduit à la mise en valeur d'organismes intermédiaires entre l'individu et l'État: corporation ou syndicat professionnel, famille, mutuelle, association. Ceux-ci doivent favoriser l'émergence d'une solidarité qui nuancerait la rigueur et l'abstraction de l'individualisme qui fonde la République. Ce qui caractérise enfin le régionalisme, pour Charles-Brun, c'est l'idée de choisir des centres régionaux suffisamment peu nombreux pour faire contrepoids à Paris, et tenant compte non seulement du poids des caractéristiques climatiques, physiques, ou culturelles traditionnelles, mais aussi des " affinités économiques nouvelles » qui se dessinent à l'aube du $\mathrm{xx}^{\mathrm{e}}$ siècle $^{14}$.

Autrement dit, le régionalisme à cette époque n'exprime pas essentiellement une position conservatrice cherchant à réintroduire un fonctionnement social d'avant 1789 , à travers la remise en vigueur des anciennes "généralités ". Même F. Le Play, par ailleurs si conservateur, n'en voulait pas. Il s'agit véritablement d'une démarche d'aménagement qui veut faire jouer un rôle à un «tiers ", qui cherche une "troisième voie » réformiste, et qui imagine la région comme lieu de structuration d'une nouvelle notabilité. Celle-ci serait du genre « conseil économique et social » ou néocorporatiste : chaque groupe social ou d'intérêt serait représenté dans une perspective solidariste compensant la rigueur du système démocratique où un homme a une voix et rien de plus ni de moins. Ceci fait penser à la composition tripartite (usagers, industriels, État) des comités directeurs d'institutions récemment créées comme par exemple nos agences financières de bassin: n'est-ce pas ce type de représentation "un groupe social, une voix » qui y est adopté ?

Il semble donc qu'un des enjeux de la régionalisation consistait depuis longtemps à profiter de l'émergence d'un échelon territorial nouveau pour faire passer une notabilité nouvelle. D'ailleurs, les régionalistes voulaient aussi une réforme communale: le groupement des communes s'accompagnerait du recul du pouvoir autocratique du maire au profit de la collégialité, qui à son tour permettrait "l'introduction de compétences techniques» dans les municipalités. Du même coup le relâchement de la tutelle serait possible ${ }^{15}$. Il y a dans ces propos quelque chose d'assez actuel...

Bien sûr, Charles-Brun ne manque pas de regretter le dépeuplement des campagnes, et de souhaiter comme Louis Marin le retour à la terre, ou comme l'abbé Lemire le redéveloppement de la petite propriété ; mais à côté de cette approche conservatrice il imagine la création d'usines à la campagne, la pluriactivité, dans une perspective qui sera reprise par la Jeunesse agricole catholique après 1945. Selon lui, l'aménagement du sol ne consiste pas seulement à protéger des pays, mais encore à mettre en valeur 
rationnellement leurs ressources (notamment énergétiques). Le reboisement des montagnes protège les plaines d'inondations et les syndicats d'initiative défendent des sites pour faciliter le développement du tourisme et donc d'une industrie hôtelière. Le régionalisme économique, ce serait un réseau de transports moins structuré en forme d'étoile ${ }^{16}$, des banques locales (comme le Crédit agricole), des Conseils régionaux du travail élus à la proportionnelle, une organisation de la protection sociale et des retraites ouvrières sur une base régionale ${ }^{17}$.

En définitive, J. Charles-Brun ne renie certainement pas le sentimentalisme d'un attachement " esthétique » et passéiste au terroir et à ses traditions, mais il fait aussi de la région le lieu d'un modernisme raisonnable appuyé sur ces considérations scientifiques. Et d'ailleurs si certaines de ses propositions paraissent tout de même bien inquiétantes, nul ne sait si elles ont été vraiment enterrées avec le régime de Vichy.

Il faut se souvenir en effet que parmi les régionalistes et les planistes (comme Justin Godard ou Louis Marin), plusieurs sont fascinés dans les années trente par une science du milieu et des races, par l'anthropométrie et la biotypologie : c'est l'époque de la montée des projets "d'hommes nouveaux", de l'attirance pour les États forts (car débarrassés du "virus de la politique » comme de la tuberculose). Et Charles-Brun comme Raymond Lizop adhéreront au régime de Vichy en espérant que la régionalisation serait le moyen de faire émerger une organisation sociale néocorporatiste offrant un meilleur rendement que la démocratie, et bien différente du passéisme folklorisant que beaucoup de Français avaient attaché au mot région dans l'entre- deux-guerres : " Tous les projets régionalistes, et, croyons-nous, celui de la commission des provinces du conseil national prévoient cette assemblée régionale : ils diffèrent sur son mode d'élection. Cependant, il est entendu généralement qu'elle n'aura pas de caractère politique et que la représentation corporative y aura sa place avec la représentation communale " (CharlesBrun, 1943). Lorsqu'on rappelle que le représentant du pouvoir central dans la région devait s'appeler le gouverneur, on a envie de faire le rapprochement avec la gestion du Maroc par H. Lyautey, celle-là même que l'on a justement réévaluée pour sa modernité (Rabinow, 1983).

22 Ayant approfondi l'étude de ce que représentait le régionalisme dans l'un de ses moments forts, au début du siècle, il nous faut reconstituer sommairement l'histoire des tentatives régionalistes et tenter d'expliquer leurs échecs.

Les avatars du régionalisme

23 Le $\mathrm{xIX}^{\mathrm{e}}$ siècle aura été le siècle de la consolidation du centralisme français, d'un jacobinisme dont la moindre des "vertus " n'était pas d'empêcher que les libertés locales retrouvées ne ressuscitent les ardeurs révolutionnaires. Il a fallu attendre longtemps pour que les réformes décentralisatrices prennent corps, et d'abord au niveau communal, puis départemental, comme le rappelle J. Charles-Brun dans un chapitre de son livre consacré à l'histoire de la décentralisation. Globalement, l'atomisation des petits possédants ruraux en fait la base du soutien aux deux empires, comme le note K. Marx dans le 18 Brumaire.

24 Dès la fin de la Commune de Paris, ce sont les radicaux qui agitent la question en imaginant que tous les niveaux territoriaux, communal, national et tous les intermédiaires, soient gérés par des conseils élus au suffrage universel. Les auteurs de la proposition, Ledru-Rollin, Brisson, Floquet sont aussi ceux qui déposent à l'Assemblée la première proposition de loi qui aboutira trente ans plus tard au vote de la loi de 1901 sur les associations. Les radicaux sont donc alors favorables à la 
recréation de niveaux intermédiaires entre l'individu et l'État. Cependant, ils n'admettent pas vraiment de remise en cause du principe de la République « une et indivisible ", ce qui limite d'emblée leur ardeur régionaliste. En particulier, ils sont sensibles à l'argument avancé par les industriels libéraux d'une égalité de tous devant la concurrence, qui serait entravée par la mise en avant de spécificités locales ou régionales, par le municipalisme social, sans même penser au socialisme municipal...

C'est surtout en 1898 que les radicaux, alors dans l'opposition, poussent à la décentralisation. C'est parce qu'ils trouvent presque partout en face d'eux un appareil territorialisé de l'État au service de leurs adversaires ${ }^{18}$. Mais dès qu'ils arrivent au pouvoir, ils la mettent entre parenthèses, et s'ils votent les vœux de $C$. Beauquier dans leurs congrès, c'est dans un silence poli. Le député du Doubs lui-même finit par dire en 1912 : "J'ai été partisan de cette réforme avec les Républicains; j'en étais parce que nous étions minorité; mais maintenant que nous sommes majorité, nous serions des niais de l'approuver. ${ }^{19} \mathrm{Au}$-delà même de l'opportunisme, la réforme régionale est un projet typiquement centriste qui n'a pas pu trouver une majorité du centre pour passer: l'affaire Dreyfus, puis la loi Combes clivent durablement «les rouges et les blancs». Puis Aristide Briand n'aura pas le temps de mettre en œuvre son projet avant la guerre. À partir du début du siècle, le thème du régionalisme va se trouver de plus en plus approprié par la démocratie chrétienne, qui va de fait le mobiliser pour combattre, ou tout au moins chercher à nuancer, le mode de représentation politique fondé sur l'élection, par la représentation corporative ou professionnelle ${ }^{20}$. De plus à droite comme La Tour du Pin aux plus à gauche comme Marc Sangnier, ils remettent en cause le libéralisme au nom du "social », et ils souhaitent au moins mâtiner les assemblées élues par des représentants (eux-mêmes élus) des groupes professionnels comme les ouvriers, les commerçants, les industriels (par «métiers»), et surtout les agriculteurs dont les organisations « régionales » leur paraissent offrir le modèle.

Dans l'entre-deux-guerres, c'est le Parti démocrate populaire, né en 1924 en réaction au Cartel des gauches, situé au centre-droit et principal antécédent du MRP en 1945, qui reprend le plus fortement le slogan régionaliste. D'autant plus qu'il recrute dans des régions frontalières ou ayant conservé de forts particularismes: Alsace, Bretagne, Savoir, Béarn...

Le contexte aurait pu être favorable à la création de régions, puisque l'organisation du ravitaillement pendant la première guerre mondiale avait légitimé une intervention désormais inévitable de l'État, et plus encore des collectivités locales, dans l'économie (Bourjol, 1975). Il en résultait un découpage régional fait en 1919 par le ministre Clémental assisté de son expert H. Hauser (régionaliste, auteur d'un projet en 1909, cf. supra), et la naissance des chambres régionales professionnelles. Le régionalisme économique était sans conteste plus actuel que le folklore. Mais les radicaux au pouvoir voulaient d'autant moins aller dans le sens d'une éventuelle acceptation de particularismes économiques que se posait avec acuité la question de la " réintégration » de l'Alsace-Lorraine à la France. On ignore aujourd'hui à quel point les mouvements régionalistes, voire l'autonomisme, y furent violents : au nom du refus de l'exploitation par les capitalistes français de ce qui devenait pour eux une colonie, les communistes alsaciens, fort nombreux, allèrent jusqu'à s'allier avec les démocrates chrétiens contre les radicaux et les socialistes ${ }^{21}$. Une part d'entre eux devait d'ailleurs rompre avec le PCF, et finir à la solde des nazis. Il serait intéressant de savoir si cette trajectoire est le fruit de la tactique, Hitler promettant une autonomie que la France 
refusait, ou si elle n'est pas aussi celui d'une fascination pour une approche en terme de milieu et de race...

Le centralisme fut donc affirmé, au détriment de cette modernité de gestion du social, dont les démocrates chrétiens alsaciens affirmaient détenir le modèle, un modèle d'origine allemand à mettre à la disposition de la France entière ${ }^{22}$. Cependant, le régionalisme économique mis en place par Clémental n'a peut-être pas été complètement inexistant. Il faudrait mieux en apprécier l'importance exacte et la liaison avec le planisme pour situer l'émergence de la DATAR dans la durée.

Dans ce contexte la région fut de plus en plus associée avec les menées antiparlementaristes, et ce n'est alors pas un hasard si elle eut son heure de gloire sous Vichy. À la Libération, à cause du rejet par contrecoup des choix de l'État français, mais aussi avec le même sens de l'opportunisme que les radicaux, le MRP, arrivé au pouvoir à la fin des années quarante, ne devait jamais mettre en application cette régionalisation qui était au cœur de sa doctrine avec la reconstitution des « corps intermédiaires » tels que la famille, le syndicat mixte, les associations... (Callot, 1978).

Sous Vichy précisément, la région devint une notion écartelée entre le traditionalisme le plus désuet et la modernité frappante de l'« équipement nationale ». Les projets du préfet régional d'Orléans, Jacques Morane, témoignent de l'ambition des technocrates qu'on a ensevelie à la Libération sous les balivernes des pétainistes condamnés à l'indignité nationale. Morane était ingénieur du corps des Ponts et Chaussées, issu d'une famille d'inventeurs d'avions. Dès 1941, il mit en place d'une part un programme de coordination des polices, et d'autre part de nouveaux échelons d'encadrement administratif des populations rurales; ceux-ci avaient notamment pour but de leur faire oublier les notables qui leur servent traditionnellement de relais ${ }^{23}$. Pour ces nouveaux fonctionnaires, il créa une école régionale d'administration. Puis il dessina les contours de l'" action économique, de l'action sociale et du régime financier de la région". $\mathrm{J}$. Morane reprenait ici la vieille idée d'une éducation populaire par la connaissance du milieu régional. Il fallait alors d'abord former les formateurs, et donc doubler l'université régionale par un institut des hautes études régionales qui redonnerait vie aux sociétés savantes. Une école régionale d'arts appliqués devait maintenir en vigueur les traditions artisanales, un conservatoire régional de musique et un centre régional d'action théâtrale devaient compléter le dispositif culturel. En matière de santé, c'est un hôpital régional qui était envisagé. Mais la région devait intervenir également dans l'économie des temps de guerre, en mettant en valeur toutes les ressources négligées jusque-là. Et pour ce faire, le préfet de région disait devoir détenir le contrôle du crédit... J. Morane voulait "instaurer un ordre qui fasse davantage appel à la fois aux techniques du progrès et à l'esprit de tradition $»^{24}$.

32 Ce projet de «Préfet-gouverneur " pour reprendre l'expression de Jacques Bardoux (1934) était trop technocratique et antidémocratique pour qu'il survive à la "Révolution nationale». Mais il est frappant par son actualité, d'autant plus que Morane était proche de plusieurs membres de l'entourage du général de Gaulle (notamment de Raoul Dautry); ceux-ci, à la Libération, conservèrent de fait le niveau territorial régional. D'autant plus surtout que l'idée d'une représentation des «forces vives ", qu'il faut bien qualifier de néocorporatisme, a bel et bien fait son chemin.

L'efficacité d'une démocratie des "intérêts »?

De ce point de vue, la IV ${ }^{e}$ République apparaît comme une parenthèse entre la fin de la $\mathrm{III}^{\mathrm{e}}$ et $\mathrm{la} \mathrm{V}^{\mathrm{e}}$, là où on voudrait voir Vichy comme parenthèse entre la $\mathrm{III}^{\mathrm{e}}$ et la $\mathrm{V}^{\mathrm{e}}$ 
République. Dès l'installation de celle-ci, les événements se précipitent, les propositions se multiplient. Michel Phlipponneau ${ }^{25}$ et Yves Mény (1971) les rappellent : Michel Debré recrée des circonscriptions d'action régionale en 1959-1960; on exhume le livre de J.-F. Gravier (Paris et le désert français, 1947) ; P. Mendès-France et le Club Jean Moulin élaborent des projets de réforme régionale (La République moderne, 1962), bientôt suivis par G. Defferre (Un nouvel horizon, 1965) et Michel Rocard (Décoloniser la province, 1966). La DATAR est créée en 1964, et avec elle les régions actuelles et les métropoles d'équilibre. Les géographes «économistes » sont mobilisés pour les analyses. Michel Phlipponneau apporte au débat l'expérience des institutions régionalisantes qu'il a menée avec d'autres en Bretagne avant de rejoindre François Mitterrand (La gauche et les régions, 1967). Vers 1968 le thème est encore activé par les propositions gaulliennes (Y.Durrien, Régionaliser la France, 1969 ; R. Lafont, La révolution régionaliste, 1967 ; J.J. Servan-Schreiber, Le pouvoir régional, 1971). La gauche non communiste est devenue régionaliste en l'espace de dix ans. On pourrait dire que ce n'est pas surprenant puisqu'elle est alors dans l'opposition et que c'est par opportunisme. Ce serait inexact, et le signe clair de la percée nouvelle du thème de la région, c'est que l'opportunisme a changé de sens. Dans les années 1950-1965, ce n'est plus la majorité conservatrice qui défend le Sénat et le statu quo entre le préfet et le conseil général, la minorité proposant une réforme décentralisatrice ou régionaliste; c'est l'opposition de gauche dans sa grande majorité qui refuse d'abandonner la pyramide de la représentation des notables du sol, par fidélité au " retour à la légalité des institutions républicaines » de 1945, et plus encore pour défendre ses bastions traditionnels. D'autant plus que la composition des $\operatorname{CODER}^{26}$ puis des Conseils économiques régionaux, dans les propositions de la majorité, fait une très large place aux élites conservatrices par le biais des membres nommés. Mais, initialement sur la défensive, la gauche modérée finit par être acquise à l'idée de composer un conseil régional pour moitié avec des élus locaux et pour moitié avec des "socioprofessionnels" au sein desquels les ouvriers auraient la majorité. C'était là fondamentalement accepter une nouvelle façon de regarder la société, au nom de l'efficacité: c'est M. Phlipponneau qui emploie le terme. Avec l'élaboration du programme commun, la gauche unie reviendra cependant à l'idée d'une assemblée régionale élue au suffrage universel direct, qui est mise en place finalement après le 10 mai 1981.

Peut-être est-ce parce que dans l'intervalle, les couches sociales qui étaient sousreprésentées dans le système politique, malgré leur importance numérique croissante depuis l'entre-deux- guerres, sont entrées beaucoup plus nombreuses dans les institutions représentatives ${ }^{27}$. Les « nouveaux notables » ont ainsi pu faire fonctionner certains conseils régionaux sur des bases nouvelles et ont accru leur légitimité par rapport à celle des conseils généraux en choisissant des thèmes d'action comme l'aménagement du territoire, l'action économique, la défense de l'environnement, qui étaient mal pris en charge par ces derniers (M. Seller in Chevallier, 1982). L'élection directe légitime désormais mieux la région. Mais comme la légitimité politique ne s'est jamais satisfaite de ce principe premier de la représentation, le bulletin de vote, il en existe toujours un deuxième, centré sur la " compétence ", c'est-à-dire sur la capacité à traiter concrètement les enjeux d'une société comme ils se cristallisent. Aujourd'hui, la compétence s'associe de plus en plus à une approche socio-économique du mode de vie salarié qui requiert toute une panoplie d'équipements adaptés aux besoins différenciés de diverses catégories de population. Il est tout à fait frappant de constater, parallèlement à la montée de cet enjeu, la progression de l'idée d'une représentation 
socioprofessionnelle de la population depuis le début du siècle : on accrédite ainsi l'idée que la société n'est pas composée d'individus atomisés, mais de groupes sociaux dont les intérêts divergent. Ce sont alors les « intérêts " qu'il faut asseoir autour d'une table par le biais de leurs représentants; ceux-ci vont alors négocier un « contrat ». Dès le début du siècle, cette conception, alors qualifiée de solidariste, s'appuie sur une représentation biologique de la société : chaque groupe social est comme un organe dont le corps social entier ne saurait se passer, mais qui a sa spécificité, ses besoins propres qu'il faudrait mesurer, négocier, satisfaire...

À son tour cette représentation biologique fait corps avec la démarche de l'étude du milieu local, qu'on appelle parfois aujourd'hui un " éco-socio-système ». La perspective globalement évolutionniste du "développement durable" cherche à combiner l'efficacité du technicien (fondée sur la maîtrise du passé et de l'avenir) et la concertation sociale. C'est l'idéal de l'aménagement du territoire. La région, entité spatio-temporelle pleine d'ambiguïtés, devait tôt ou tard être saisie par cette démarche.

Un exemple concret de cette évolution est offert par l'émergence, dans la plupart des pays européens, d'institutions régionales de gestion de la ressource en eau : que leur territoire corresponde aux bassins-versants, ou qu'il suive les régions administratives, la tendance est que leurs conseils d'administration, leurs boards, soient composés de représentants des divers « intérêts » en présence (industriels, agriculteurs, compagnies d'électricité, consommateurs domestiques, pêcheurs, écologistes...) et non pas d'élus au suffrage direct. En France en particulier, les élus locaux présents aux conseils d'administration des agences de l'eau y sont en tant que représentants des particuliers de leur commune (Barraqué, 1992).

Pour aller plus loin dans cette recherche, il semblerait nécessaire de se pencher maintenant sur la façon même dont cette étude du milieu local, qui a servi à légitimer la région, devait être conduite aux yeux de ses promoteurs. Nous avons en effet découvert l'existence de la Société des études locales, dans un ouvrage consacré à la pédagogie active par l'étude du milieu. L'auteur était un disciple de l'Écossais Patrick Geddes, l'un des grands pionniers de l'urbanisme. Or Geddes revendiquait d'avoir transformé le civic survey ou l'enquête sociale et urbaine, en regional survey, car il considérait comme indispensable de replacer l'étude de la cité dans celle de son environnement, tout en faisant conduire l'enquête par les citadins eux-mêmes. Cette perspective de pédagogie active fonde l'espoir d'une participation accrue des citadins à la vie publique sur la connaissance par ceux-ci de leur milieu physique et social d'existence.

Or Patrick Geddes était lui-même inspiré par les travaux d'Auguste Comte et de Frédéric Le Play, et d'ailleurs le "Collège des Écossais » qu'il avait créé à Montpellier s'intitulait « Comte-Le Play-Geddes ». Ses liens avec la France étaient donc multiples. Il fut l'ami d'Élisée Reclus et de Marcel Poëte, et sans doute de bien d'autres géographes et urbanistes français comme d'autre pays d'Europe. Il serait donc intéressant de creuser cette piste de la pédagogie active par l'étude du milieu dans son contexte international. Cela permettrait peut-être de mieux comprendre pourquoi en France, la tentative de la Société des études locales est restée mineure sur le moment, mais aussi avec quelle force, progressivement, cette vision plus « organiciste » de la société et de son rapport au territoire s'impose comme pilier de la légitimité politique. 


\section{BIBLIOGRAPHIE}

AUZELLE R. et GOHIER H., 1963, 323 citations sur l'urbanisme, Paris, Vincent et Fréal.

BARDOUX M., 1934, Le drame français, Paris, Ed. des portiques.

BARKER M., 1931, L'utilisation du milieu géographique à des fins pédagogiques, Paris, Flammarion.

BARRAQUE B., 1991, La source du Lizon, Revue juridique de l'environnement, IV.

BARRAQUE B., 1992, Water management in a few European countries, Flux, CNRS/GDR 903, I.

BARRES M., 18974, De Hegel aux cantines du Nord, La Cocarde.

BERDOULAY V., 1981, La formation de l'école française de géographie, 1870-1914, Paris, Bibliothèque nationale, Comité des Travaux historiques et scientifiques.

BOUNEAU C., 1986, Contribution à l'histoire des régions françaises : Hubert Lagardelle et l'émergence d'une région économique du sud-ouest dans l'entre-deux-guerres, Revue économique du Sud-ouest, 2.

BURLEN K. (sous la dir. de), 1987, La banlieue-oasis, Henri Sellier et les cités-jardins, Paris, P.U. de Vincennes.

BOURJOL M., 1975, La réforme municipale, Paris, Berger-Levrault.

CALLOT E.F., 1978, Le mouvement républicain populaire, Paris, Marcel Rivière.

CHARLEES-BRUN J., 1911, Le régionalisme, Paris, Bloud.

CHARLES-BRUN J., 1943, Le régionalisme, Cahiers de formation politique, $\mathrm{n}^{\circ} 14$, Vichy.

CHEVALLIER J. et alii, 1982, Le pouvoir régional, Paris, PUF-GRAL.

GROS-MAYREVIELLE F., 1907, De la protection des monuments historiques ou artistiques, des sites et des paysages, thèse, Université de Paris.

DUMAZEDIER J., 1967, Vers une civilisation des loisirs, Paris, Le Seuil.

DUMAZEDIER J., SAMUEL N., 1976, Société éducative et pouvoir culturel, Paris, Le Seuil.

GAUDIN J.-P., 1986, Pouvoirs locaux et territoires, Études rurales.

GAUDIN J.-P., 1990, Technopolis, crises urbaines et innovations municipales, Paris, PUF, coll.

«Économie en liberté ».

GEDDES P., 1949, Cities in evolution (Edimbourg, 1908), Londres, Williams et Norgate Ltd.

GRAS C., LIVET G. (sous la dir. de), 1977, Régions et régionalisme en France, Paris, PUF.

GREMION P., 1976, Le pouvoir périphérique, bureaucrates dans le système politique français, Paris, Le Seuil.

GUICHARD O., 1976, Vivre ensemble, Paris, La Documentation française.

LIZOP R., 1938, Pourquoi le régionalisme, Toulouse, Ed. de l'archer.

MARIE M., 1986, Penser son territoire, in AURIAC F., BRUNET R. (édis.), Espace, jeux et enjeux, Paris, Fayard.

MARIE M. et alii, Cahiers Territoires, techniques et sociétés, diffusés par la Délégation à la recherche et à l'innovation du MELTE. 
MENY Y., 1971, Centralisation et décentralisation dans le débat politique français, 1945-1969, thèse, Université de Rennes.

Ministère de l'Instruction publique et des Beaux-arts, circulaire du 25 fév. 1911, Bull. admin., 11 mars $1911, \mathrm{n}^{\circ} 1970$.

POINSOT (Chanoine), 1907, Les vraies bases du régionalisme, Les pages modernes.

RABINOW P., 1988, Biopower in the colonies (CNRS Lyon, 1983), trad. fr. B. Barraqué in Colonie et métropole, Dossiers T.T.S. de la DRI du MELTE, $\mathrm{n}^{\circ} 4$.

ROBIC M.-C., 1989 a, « Métropole/métropole, les géographes et les métropoles d'équilibre », Strates, 4, CNRS/URA 142, p. 67-88.

ROBIC M.-C., 1989 b, « Perspectives temporelles sur l'émergence de quelques concepts de la géographie urbaine française », Sistemi urbani, 3, p. 225-239.

VIDAL DE LA BLANCHE P., Régions françaises, Revue de Paris, 15 déc. 1910.

\section{NOTES}

1. Ce texte reprend une contribution personnelle au séminaire Aménagement et ménagement des territoires organisé par M. MARIE, B BARRAQUE et V. CLAUDE à la Délégation à la recherche et à l'innovation du ministère de l'Urbanisme, du Logement et des Transports en 1985-1986. Il s'agissait de repenser un certain nombre de notionsclés de l'aménagement, de faire l'état de la question, et de relancer la recherche en provoquant des discussions. C'est ainsi qu'on a travaillé sur des séquences comme " colonies et métropoles ", " réseaux et modèles d'organisation spatiale ", " régions ", " rénovation-réhabilitation »... Du point de vue méthodologique, il s'agissait à travers ces objets de développer la réflexion en trois temps : - l'aménagement comme imposition de la rationalité centralisatrice aux périphéries ; - le ménagement comme résistance du local au central ; - et au-delà, le débat entre les deux comme révélateur du changement du regard porté par la société sur l'espace et sur elle-même (cf. MARIE et alii).

2. Si le département paraît lié au jacobinisme, c'est qu'il est à la fois une structure démocratique qui permettait aux couches populaires rurales d'être représentées, mais qu'en même temps le rôle très important du préfet permettait aux élites capitalistes de dominer les petits possédants : au centre la technicité, à la périphérie la ruse locale, l'adaptation de la norme technique. P. GREMION remarque cependant que son modèle de pouvoir périphérique est battu en brèche dans les villes les plus grandes, dans la mesure où le partage des tâches départementales entre « le préfet et les notables » n'est plus de mise. De même, J. CHEVALLIER dans Le pouvoir régional (PUF), 1983, montre-t-il la montée de la région comme institution administrative au détriment des départements.

3. $C f$. Circulaire du ministère de l'Instruction publique et des Beaux-arts du 25/02/1922, in Bull. Admi. 11/03/ 1911 nº $^{\circ} 1970$; et J. CHARLES-BRUN, 1911.

4. À la bibliothèque nationale, car le ministère de l'Éducation nationale n'a pas de bibliothèque où l'on puisse consulter ses archives - en particulier les numéros anciens de sa propre revue, la Revue pédagogique. Celle-ci contient dans sa livraison de 1911 un article d'Edmond BLANGUERNON, « L'école et la région », introuvable aujourd'hui.

5. Circulaire du 25/02/1911, op. cit. 
6. Cf. B. BARRAQUE, in BURLEN, 1987 ; à la Libération, on a tenté de créer les « classes nouvelles » où l'étude du milieu local était en bonne place. Edmond DEMOLINS, disciple moderniste de F. LE PLAY, était inspiré par l'expérience de Patrick GEDDES lorsqu'il créa l'école des Roches.

7. P. VIDAL DE LA BLACHE, 1910. Il proposait de redécouper la France en 17 régions relativement spécialisées au plan économique.

8. Projets BECHARD (1851), RAUDOT (1851), Auguste COMTE (1854), F. LE PLAY (1864), FOURNIER DE FLAIX (1869), Hervé BAZIN (1886), F. LEPELLETIER (1896), P. FONCIN (1898), G. SORTAIS (1902), CORDIER-JOLY (1903), L. SENTUPERY (1903), LieutenantColonel ROYAL (1906), Maurice TOUSSAINT (1906), Henri BARRE (1906), de la TOUR DU PIN et CHAMBLY (1906), Henri MAZEL (1907), L'HUILLER (1907), B. SARRIEU (1907), J.B. RUFFIN (1908), J. FEVRE et H. HAUSER (1909), P. VIDAL DE LA BLACHE (1910); et RAUDOT (1851), HOVELACQUE (1890), de LANJUINAIS, de RAMEL et alii (1895), de CORNUDET et LOCKROY (1896), de RAMEL et MACKAU (1898), Louis MARTIN et CHASSAING (1901), Charles BEAUQUIER (1902). On remarquera l'absence de projets pendant les quinze années qui ont fait suite à la Commune de Paris. Cf. J. CHARLESBRUN, op. cit., appendice $\mathrm{n}^{\circ}$ III.

9. Le félibréen BERLUC-PERUSSIS.

10. Avec Paul BOURGET, de LANJUINAIS, de MARCERE, etc.

11. Le Comité d'honneur comprenait Paul ADAM, Jean BAFFIER, Maurice BARRES, $P$. BAUDIN, Antide BOYER, Dr BUCHER, CLOAREC, M. COLIN, Paul DESCHANEL, Paul DOUMER, de l'ESTOURBILLON, P. FONCIN, Eug. FOURNIERE, de GAILLARD-BANCEL, J. GODARD, André HALLAYS, HEMON, Vincent d'INDY, KRANTZ S-C LECONTE, Jules LEGRAND, Abbé LEMIRE, Louis MARIN, A. METIN, P. MOREL, F. MISTRAL, J.PAULBONCOUR, A. RIBOT, A. de la ROCHEFOUCAULD, Maurice SCHWOB. Plusieurs de ces personnes sont parmi les fondateurs de la Société pour la protection des paysages de France : André HALLAYS, CLOAREC, J. BAFFIER, LE GOFFIC. Plusieurs sont des défenseurs des sites des Côtes du Nord. Louis MARIN est député de Nancy, professeur de " méthodes ethniques et sociales » au Collège libre des sciences sociales. P. FONCIN était un géographe auteur d'un projet de découpage régional.

12. Cf. J. CHARLES-BRUN, Le Régionalisme, op. cit., ch. III.

13. Cf. C. POINSOT, Les vraies bases du régionalisme, in Les pages modernes, 1907. Ces bases sont une sorte de darwinisme appliqué aux races et aux groupes humains.

14. La place importante accordée à la capitale régionale rapproche l'auteur de VIDAL DE LA BLANCHE, qui, lui aussi, associe région et métropole régionale (à la fois industrielle, bancaire, culturelle). Cf. ROBIC, 1989 b.

15. J. CHARLES-BRUN, Le Régionalisme, op. cit., ch. V.

16. Il est notamment question de l'axe Lyon-Bordeaux à travers le Massif Central.

17. J. CHARLES-BRUN, op. cit., ch. VII, Régionalisme économique et social.

18. Cf. S. BERSTEIN, Le Parti radical et le problème du centralisme, in Régions et Régionalisme en France, actes du colloque, réunis par C. GRAS et G LIVET, Paris, PUF, 1977, p. 228.

19. Ibid., p. 232

20. Cf. J.M. MAYEUR, Démocratie chrétienne et régionalisme, in GRAS et LIVET, op. cit.

21. Cf. B. REIMERINGER, Un communisme régionaliste, le communisme alsacien, in GRAS et LIVET, op. cit., p. 337-361.

22. F.G. DREYFUS, Le général de Gaulle et la régionalisation, in GRAS et LIVET, op. cit., p. 576. 
23. Y. DURAND, La politique régionale de Vichy dans la pratique, in GRAS et LIVET, op. cit., p. 507-527.

24. J. MORANE, 30/03/1942, cité par Y. DURAND in GRAS et LIVET, op. cit.

25. M. PHLIPPONNEAU, La gauche et le régionalisme, in GRAS et LIVET (1977), op. cit., p. 529-543.

26. Commissions de développement économique régional.

27. Voir à ce sujet les analyses des chercheurs en sciences politiques comme

C. SORBETS, J. PALARD à Bordeaux ou J. CHEVALLIER, P. LEHINGUE à Amiens.

\section{RÉSUMÉS}

La région, appelée à prendre de l'importance dans le cadre de l'Europe communautaire, est une entité à bien des égards floue, revendiquée qu'elle a été d'un côté par les partisans d'un aménagement rationnel du territoire, et d'un autre côté par les défenseurs d'un patrimoine local. Le recours à l'histoire du régionalisme et des « études locales »depuis le début du siècle permet de montrer qu'au cœur même du débat entre les deux types de protagonistes, s'esquisse une nouvelle manière pour la société de se représenter elle-même et donc de définir sa légitimité politique.

Region, regionalisms and planning

In France, the region, which will play an important role in the development of post 1993-Europe, is still an imprecise notion. Some wanted to use it as the basis for a modern regional planning, others on the contrary identified it with the defense of an heritage threatened by modernization. A history of regionalism and «local surveys " in the early $20^{\text {th }}$ century allows to show that the very debate between modernization and conservation brings up a new way for society to look at itself and in define its political legitimacy.

\section{INDEX}

Mots-clés : Aménagement, régions, régionalismes, études locales, notabilité, légitimité politique, xxe siècle

Keywords : France, region, regionalisms, local surveys, notability, political legitimacy, xxth

\section{AUTEUR}

\section{BERNARD BARRAQUE}

Chargé de recherches au CNRS, rattaché au laboratoire Techniques, Territoires et Sociétés, il travaille actuellement à une comparaison des institutions de gestion de l'environnement, et en particulier de l'eau, dans les pays de la CEE. 